Research Paper

\title{
Interleukin 37 Expression Inhibits STAT3 to Suppress the Proliferation and Invasion of Human Cervical Can- cer Cells
}

\author{
Sen Wang1,\#, ${ }^{凶}$, Weifang An1,\#, Yunhong Yao1,\#, Renhuai Chen ${ }^{1}$, Xiaoxuan Zheng1,2, Wanyong Yang ${ }^{2}, Y_{i}$ \\ Zhao $^{1}$, Xinrong $\mathrm{Hu}^{1}$, Enping Jiang ${ }^{1}$, Yanhong Bie ${ }^{3}$, Zhangquan $\mathrm{Chen}^{1}$, Ping Ouyang ${ }^{1}$, He Zhang ${ }^{1}$, Hui \\ Xiong1 \\ 1. Cancer Institute, Guangdong Medical University, Dongguan 523808, China; \\ 2. Clinical Laboratory, Guanghua hospital, Dongguan 523808, China; \\ 3. Pathology, People's Hospital of Xiaolan, Zhongshan 528415, China. \\ \# The three authors contribute equally to this work and are co-first authors. \\ $\square$ Corresponding author: Sen Wang, Address: Cancer Institute of Guangdong Medical University, No. 1 Xincheng Road, Dongguan, Guangdong \\ Province 523808, PR China. Tel: +86 769 22896401; Fax: +86 769 22896401; E-mail: wangsen@gdmc.edu.cn wangsenmol@163.com
}

(c) 2015 Ivyspring International Publisher. Reproduction is permitted for personal, noncommercial use, provided that the article is in whole, unmodified, and properly cited. See http://ivyspring.com/terms for terms and conditions.

Received: 2015.03.28; Accepted: 2015.07.20; Published: 2015.08.08

\begin{abstract}
Objectives: The most recently discovered cytokine interleukin 37 (IL-37) received growing attention. Its function on tumor is largely unknown. Here, we investigated the biological function of IL-37 on cervical cancer (CC).

Materials and methods: $\mathrm{HPV}^{+}$Hela cells and HPV ${ }^{-} \mathrm{C}^{3} \mathrm{~A}$ A cells were used. RT-qPCR was performed to detect the transcription of IL-37, STAT3, TNF- $\alpha$ and IL-1 $\beta$. Western blotting was used for protein detection. CCK-8 assay and transwell assay were employed for cell proliferation and invasion detection, respectively.

Results: Successful gene transfection of IL-37 suppressed the proliferation and invasion of CC. Interestingly, IL-37 showed higher anticancer ability in $\mathrm{HPV}^{+}$Hela cells than that in HPV $\mathrm{C}^{-} \mathrm{C} 3 \mathrm{~A}$ cells. Then, the molecular mechanism of IL-37 anticancer was explored. Firstly, we found that IL-37 inhibited STAT3 expression at both mRNA and protein levels. IL-37 also down regulated the phosphorylation of STAT3. Secondly, blockage of STAT3 using siRNAs reduced significantly the ability of IL-37 to suppress cell proliferation and invasion. Thirdly, STAT3 knockdown reduced markedly the inhibition of IL-37 on the transcription of tumor-derived TNF- $\alpha$ and IL-1 $\beta$, indicating the contribution of STAT3 for the cancer associated antiinflammation of IL-37. Finally, STAT3 up regulation restored the ability of cell proliferation, cell invasion and the expression of inflammatory cytokines, TNF- $\alpha$ and IL- $1 \beta$.

Conclusions: IL-37 suppressed cell proliferation and invasion of CC and STAT3 is involved in this process. Thus, IL-37 emerges as a new anticancer cytokine for CC. This study demonstrated a new biological function of IL-37 and offered a potential molecule for CC treatment.
\end{abstract}

Key words: Interleukin 37; Cervical cancer; Proliferation; Invasion.

\section{Introduction}

The newly named cytokine interleukin 37 (IL-37) has been identified as an inhibitor of both innate and adaptive immunity. The novel gene is the seventh member of IL-1 family (IL1F7) and was renamed IL-37 in 2010. It is located on chromosome 2 and contains six exons that encode a 17-26KD protein. Recent studies report that IL-37 shows anti-inflammatory effects in colitis [1], liver inflammatory injury [2], 
contact hypersensitivity [3], and other diseases [4-8]. Importantly, in 2010, Nold et al [9] discovered that IL-37 and Smad3 form a functional complex and smad3 is required for IL-37 activity. In their experiment, smad3 knockdown reduces the activity of IL-37. In 2014, our group found that dendritic cells expressing IL-37 are tolerogenic. They induced Treg cells and impaired the activation of effector T-cell responses in contact hypersensitivity [3]. However, most of these studies focused mainly on the inflammatory diseases, the requirements for IL-37 exploration is capturing more and more attention.

A number of IL-37 mysteries remain. At the gene level, although IL-37 has been demonstrated to function partly through IL-37-smad3 complexes in the nuclei, it is not known whether there are other molecules that function the maximum regulation on IL-37. At the molecular level, although IL-37 could regulate downstream signal pathways in many immune related cells, it is not known clearly whether this is the case in non-immune cells. At the disease level, although IL-37 has been a useful inhibitor of immunity, it is not known completely whether IL-37 has similar powerful capabilities in other diseases, such as cancer. Moreover, does IL-37 influence virus and bacterium that induce the body inflammation and how? Does IL-37 have a role in the cell-cell interactions and what is it? For IL-37, challenges and opportunities coexist.

The function of IL-37 in tumor is largely unknown. Inflammation is the seventh hallmark of tumors [10], inferring that IL-37 might influence inflammation-related tumors. A very limited numbers of studies have suggested that IL-37 has anticancer ability. In 2003, Gao et al [11] reported that fibrosarcoma growth was suppressed by injection of IL-37 in mice. In 2014, Zhao et al [12] found that IL-37 expression was decreased in human hepatocellular carcinoma (HCC) tissues and IL-37 might play an important role for the prognosis of HCC patients. Cervical cancer (CC) is the second most common cancer and is the fifth leading cause of cancer death in women [13]. CC relates to human papillomavirus (HPV) infection and the immunological status of the body. Chronic inflammation is of critical importance in the CC progress, suggesting that IL-37 might have a role in CC. However, the function of IL-37 on CC remains elusive. Here, we offer firstly the evidences that IL-37 suppresses the proliferation and invasion of CC cells and signal transducer and activator of transcription 3 (STAT3) is involved in the process.

\section{Materials and Methods}

\subsection{Materials}

Anti-IL-37 antibody (1:500), anti-STAT3 anti- body (1:500) and anti-pSTAT3 antibody (1:500) were bought from Abcam, Cambridge, UK. Hilymax (Dojindo, Shanghai, China) was used for gene transfection. STAT3 siRNAs (siSTAT3) were from Genepharm Co, Ltd, Shanghai, China. Cell Counting Kit-8 (CCK-8) was from Sangon, Shanghai, China.

\subsection{Cell culture and gene transfection}

Two CC cell lines, $\mathrm{HPV}^{+}$Hela and HPV- C33A were used. The cells were cultured in the standard medium (RPMI 1640 containing 10\% FBS, 2 mmol/L l-glutamine, $50 \mathrm{U}$ penicillin and $50 \mu \mathrm{g} / \mathrm{ml}$ streptomycin) at $37^{\circ} \mathrm{C}$ in $5 \% \mathrm{CO} 2$-in-air. IL-37 expression vector (IL-37-pIRES2-EGFP) was constructed ourselves. STAT3/pcDNA3.1 expression vector were bought from Dongguan Yecam Biotech Co., LTD. The sequence of STAT3 siRNA was previously published and had been proved to be effective in our pre-tests [14]. The IL-37 plasmids or siRNA of STAT3 were transfected into cellls using $5 \mu$ l Hilymax (Dojindo, Shanghai, China) for per $\mu$ g DNA. HilyMax is a newly developed gene transfection reagent that forms a liposome to be used for highly efficient gene transfection to a wide variety of cells. When transfection, $120 \mu \mathrm{l}$ serum-free medium, $3.0 \mu \mathrm{g}$ plasmid DNA and $15 \mathrm{uL}$ Hilymax were added to the tube and incubated at room temperature for 15 minutes to form DNA-Hilymax complex. At last, the complex was added to the cells.

\subsection{RT-qPCR detection}

Primer sequences were as follows, forward: $5^{\prime}-$ TGTGCGTATGGGAACACCTA-3', reverse: 5'-AGA AGGTCGTCTCCCCCTTA-3' for STAT3; forward: 5'-GATCACAAAGTACTGGTCCTGG-3', reverse: 5'-TCCTTTATCCTTGTCACAGTAG-3' for IL-37; forward: 5'-GAAGGTCGGAGTCAACGGATTT-3', reverse: 5'-CCTGGAAGATGGTGATGGGATT-3' for GAPDH. Reverse transcription (RT) was performed using the RT Kit (Takara, Dalian, China) according to the manufacturer's protocols on the GeneAmp PCR System 9700 (ABI, USA). For IL-37 and STAT3 amplification, $0.05 \mu \mathrm{g}$ of total RNA was used in 20 $\mathrm{\mu l}$ RT volume. Quantitive PCR (qPCR) was performed on the 7500 Real-Time PCR ABI system (ABI, USA), using a 96-well plate format. PCR was carried out in triplicate in a $20 \mu \mathrm{L}$ reaction volume containing $10 \mu \mathrm{l}$ Platinum_® SYBR®Green (Invitrogen, Guangzhou, China), $0.8 \mu \mathrm{l}$ forward primer $(10 \mu \mathrm{M}), 0.8 \mu$ l reverse primer $(10 \mu \mathrm{M}), 1 \mathrm{uL}$ cDNA and $7.4 \mathrm{uL} \mathrm{ddH}_{2} \mathrm{O}$. The reaction conditions were $95^{\circ} \mathrm{C}$ for $10 \mathrm{~min}, 40$ cycles of $95^{\circ} \mathrm{C}$ for $15 \mathrm{~s}$ and $60^{\circ} \mathrm{C}$ for $1 \mathrm{~min}$ [15]. Data were analyzed using the sequence detection software version 1.6.3 supplied by Applied Biosystems (ABI, USA). 


\subsection{CCK-8 cell proliferation assay}

Cell proliferation was determined by Cell Counting Kit-8 (CCK-8) according to the manufacturer's protocol (Sangon, Shanghai, China). CCK-8 allows convenient assays using reagent WST-8, which is bio-reduced by cellular dehydrogenases to an orange formazan product. The amount of formazan produced is directly proportional to the number of living cells. Cell suspension was inoculated in a 96-well plate (104/well). $10 \mu \mathrm{l} \mathrm{CCK-8} \mathrm{solutions} \mathrm{were}$ added to each well and the incubation was continued
A
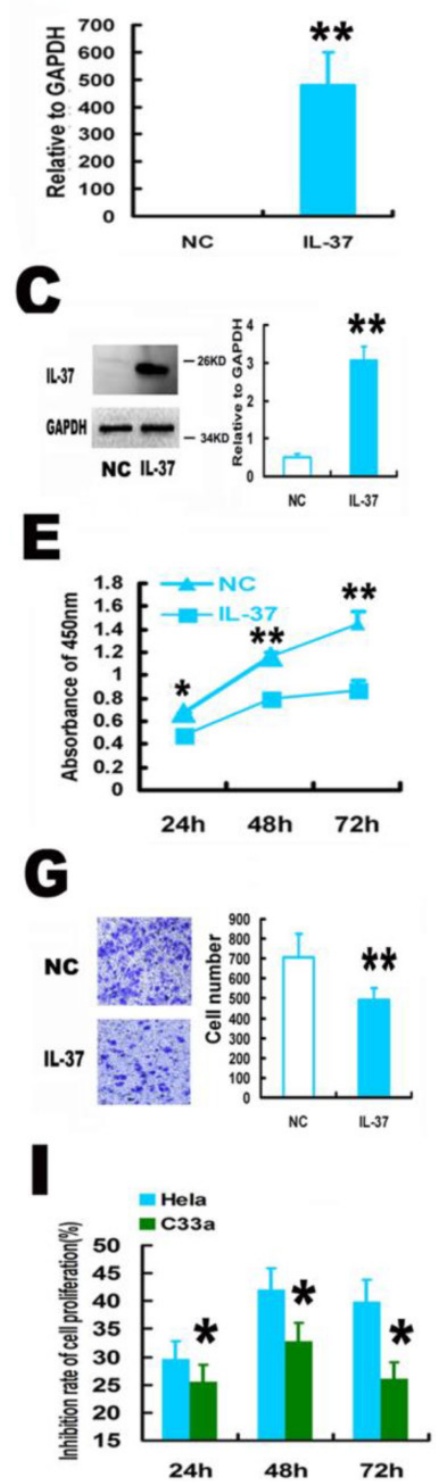

B

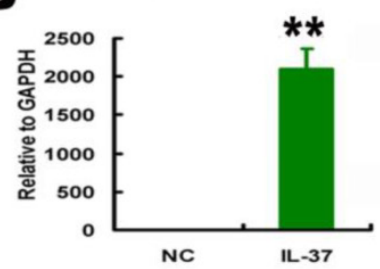

D

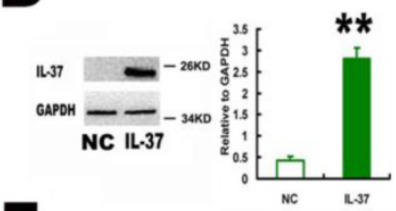

$\mathbf{F}$

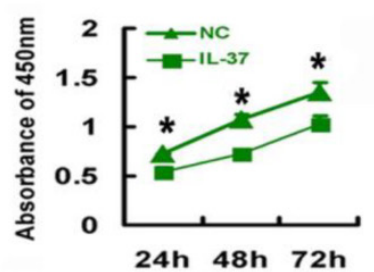

H

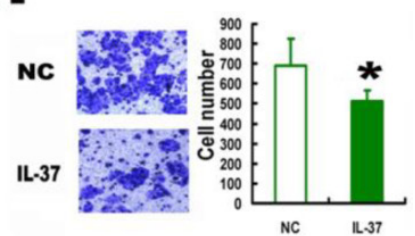

$\boldsymbol{J}$

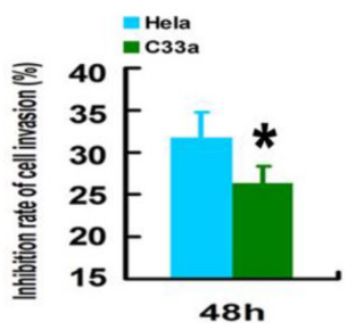

Figure 1. Expression of human IL-37 suppressed the proliferation and invasion of HPV+ $\mathrm{Hela}$ and HPVC33A cells. (A, B) Transfection of IL-37 increased the mRNA expression of IL-37 in Hela (A) and C33A (B) cells. (C, D) Transfection of IL-37 increased the protein expression of IL-37 in Hela (C) and C33A (D) cells. $(E, F)$ IL-37 inhibited the cell proliferation of Hela $(E)$ and C33A (F) cells. $(G, H)$ IL-37 suppressed the cell invasion of Hela $(\mathrm{G})$ and $\mathrm{C} 33 \mathrm{~A}(\mathrm{H})$ cells. (I) IL-37 showed higher anticancer ability to suppress cell proliferation in $\mathrm{HPV}^{+} \mathrm{Hela}$ cells than that in HPV-C33A cells. (J) IL-37 showed higher anticancer ability to suppress cell invasion in $\mathrm{HPV}^{+}$Hela cells than that in HPV- $\mathrm{C} 33 \mathrm{~A}$ cells. $(\mathrm{N}=3)$. NC: normal control gourp (transfection of blank expression vector). IL-37: IL-37 gene transfection group. for additional $2 \mathrm{~h}$. The experiment was done in five wells per group. The absorbance (A) for the wells was tion rate were calculated: Inhibition rate $(\%)=$ (1-Atreated group/Amock) $\times 100 \%$ [15]

Invasive ability of cells was determined within a transwell system. $6.0 \times 10^{4}$ cells were vaccinated into cultured at $37^{\circ} \mathrm{C}$ in $5 \% \mathrm{CO}_{2}$-in-air for $24 \mathrm{~h}, 48 \mathrm{~h}$ and $72 \mathrm{~h}$. The number of cells that migrated to the lower surface of the membrane was counted under a microscope (200×) [15].

\subsection{Statistical analysis}

Statistical software SPSS13.0 was used to perform the statistical analysis. Data were reported as means \pm standard deviation (SD) .Independent samples $t$ test was used for analyzing two groups and one way analysis of variance (ANOVA) was used for examining multiple groups. Rates were compared using chi-square test. The level of statistical significance was set at $\mathrm{P}<0.05$ or $\mathrm{P}<0.01$.

\section{Results}

\subsection{IL-37 expression suppressed the proliferation and invasion of Hela and C33A cells}

The function of IL-37 on CC is unknown. Here, human IL-37 expression was performed in CC cells by gene transfection.

IL-37 gene transfection induced successfully IL-37 expression in both Hela and C33A cells. IL-37 expression induced significant increase at IL-37 mRNA level, $700 \%$ and $2500 \%$ for Hela cells (Figure 1A) and C33A cells (Figure 1B), respectively. No clear protein bands were detected in normal cells by western blotting while thick electrophoretic bands of IL-37 proteins were found in cells of IL-37 transfection group (Figure $1 \mathrm{C}$ and Figure 1D). IL-37 gene expressed a $25 \mathrm{KD}$ protein in CC cells (Figure $1 \mathrm{C}$ and Figure 1D), which is similar to that in A549 cells and RAW cells [9].

IL-37 expression suppressed cell proliferation and invasion. IL-37 expression suppressed the cell proliferation by $29.1 \%$ (24h), $41.0 \%$ (48h), $40.2 \%$ 
(72h) for Hela cells (Figure 1E) and by $24.9 \%$ (24h), $33.1 \%$ (48h), 24.1\% (72h) for C33A cells (Figure 1F). The cell invasion was also reduced by $30.39 \%$ for Hela cells (Figure 1G) and $26.09 \%$ for C33A cells at 48h (Figure 1H).

Interestingly, IL-37 showed higher inhibition rates of cell proliferation (Figure 1I) and cell invasion (Figure 1J) in HPV+ Hela cells than that in HPV- C33A cells. These results suggested that the anticancer ability of IL-37 might relate to virus infection and this novel gene might have a unique role in viral diseases.

\subsection{IL-37 inhibited the expression and phos- phorylation of STAT3}

IL-37 expression inhibited STAT3 by $35.1 \%$ for Hela (Figure 2A) and by $39.0 \%$ for C33A cells at mRNA level (Figure 2B), and by 32.1\% for Hela (Figure $2 \mathrm{C}$ ) and by $23.5 \%$ for C33A cells at protein level (Figure 2D). IL-37 inhibited the phosphorylation of STAT3 by $36.4 \%$ for Hela (Figure 2C) and by $30.0 \%$ for C33A cells (Figure 2D).

\subsection{STAT3 knockdown reduced the ability of IL-37 to suppress the cell proliferation and in- vasion}

In order to determine whether STAT3 is involved in the anticancer process of IL-37, siRNA of STAT3 (siSTAT3) was used.

siSTAT3 successfully knockdown STAT3 expression. The mRNA level of STAT3 was reduced by
$85.1 \%$ in Hela cells (Figure 3A) and 79\% in C33A cells (Figure 3B). The protein level of STAT3 was reduced by $55.6 \%$ in Hela cells (Figure 3C) and $61.98 \%$ in C33A cells (Figure 3D), respectively. Figure $3 \mathrm{E}$ and $3 \mathrm{~F}$ showed that siSTAT3 and IL-37 gene transfection suppressed the cell proliferation. Figure $3 \mathrm{G}$ and $3 \mathrm{H}$ showed that siSTAT3 and IL-37 gene transfection suppressed the cell invasion.

After trasnfection with STAT3 siRNA (siSTAT3), the ability of IL-37 to suppress cell proliferation and invasion was markedly reduced. In Hela cells, under the state of only IL-37 treatment, the proliferation inhibition rates of cells were $29.1 \%$ (24h), $41.0 \%$ (48h) and $40.2 \%$ (72h). While under the state of STAT3 knockdown, the proliferation inhibition rates by IL-37 expression were reduced to $13.3 \%$ (24h), $17.6 \%$ (48h) and $17.2 \%$ (72h) (Figure 3I). In C33A cells, under the state of only IL-37 treatment, the proliferation inhibition rates of cells were $24.9 \%$ (24h), $33.1 \%$ (48h) and $24.1 \%$ (72h). While under the state of STAT3 knockdown, the proliferation inhibition rates by IL-37 expression were reduced to $15.4 \%$ (24h), $14.1 \%$ (48h) and $9.9 \%$ (72h) (Figure 3J).

The inhibition rates of invasion were reduced from $30.39 \%$ to $13.11 \%$ for Hela cells (Figure 3K) and from $26.09 \%$ to $12.22 \%$ for C33A cells at $48 \mathrm{~h}$ (Figure 3L). These results inferred that STAT3 knockdown reduced the antitumor ability of IL-37.
$\mathbf{A}$

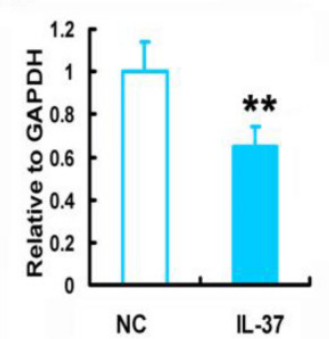

B

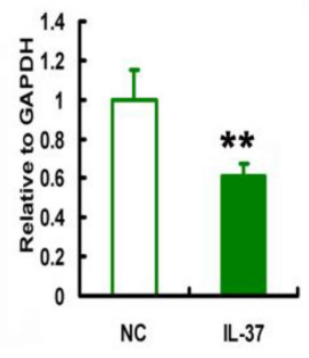

C

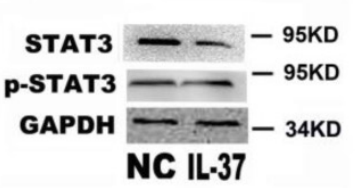

D

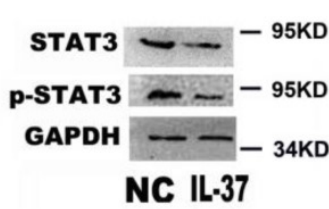

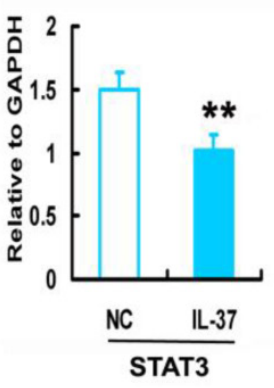
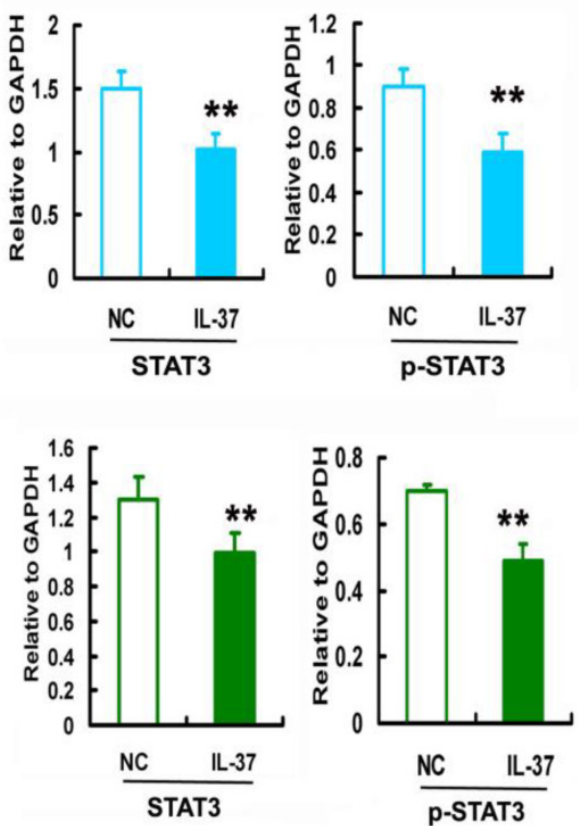

Figure 2. IL-37 suppressed STAT3 expression in cervical cancer cells. (A) IL-37 suppressed the mRNA expression of STAT3 in Hela cells. (B) IL-37 suppressed the mRNA expression of STAT3 in C33A cells. (C) IL-37 suppressed the protein expression and phosphorylation of STAT3 in Hela cells. (D) IL-37 suppressed the protein expression and phosphorylation of STAT3 in C33A cells. NC: normal control gourp (transfection of blank expression vector). IL-37: IL-37 gene transfection group. 
A

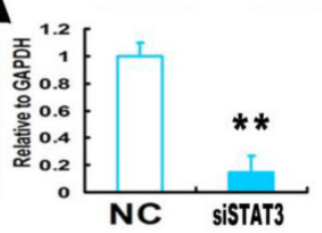

C

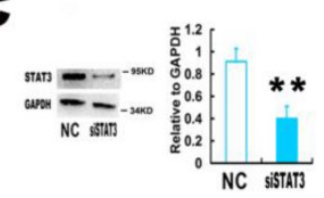

$\mathbf{E}$

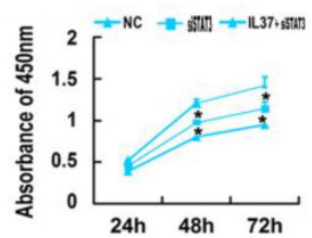

B

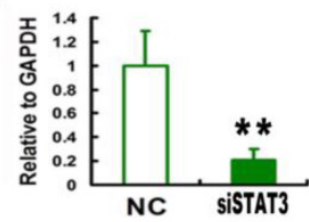

D

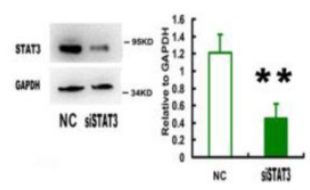

$\mathbf{F}$

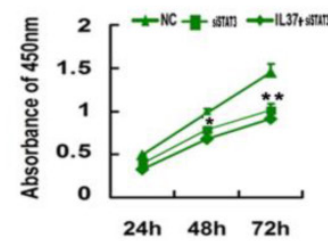

G
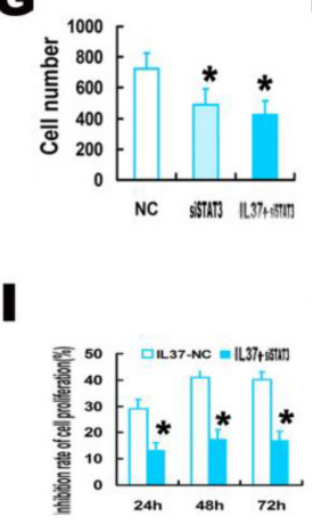

J

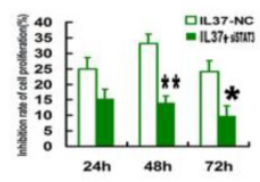

$\mathbf{K}$

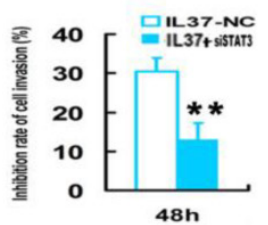

Figure 3. STAT3 siRNA (siSTAT3) transfection reduced the anticancer ability of IL-37 in Hela and C33A cells. (A, B) SiSTAT3 suppressed the mRNA expression of STAT3 in Hela (A) and C33A (B) cells. (C, D) siSTAT3 suppressed the protein expression of STAT3 in Hela (C) and C33A (D) cells. (E, F) siSTAT3 and IL-37 transfection suppressed the cell proliferation of Hela (E) and C33A (F) cells. (G, H) siSTAT3 and IL-37 transfection suppressed the cell invasion of Hela (G) and C33A (H) cells. (I, J) STAT3 siRNA transfection reduced the anticancer ability of IL-37 to suppress cell proliferation of Hela (I) and C33A (J) cells. (K, L) STAT3 siRNA transfection reduced the anticancer ability of IL-37 to suppress cell invasion of Hela (K) and C33A (L) cells. NC: normal control. siSTAT3: STAT3 knockdown group. IL37-NC: IL-37 gene transfection group. IL37+siSTAT3: IL-37 gene transfection with blockage of STAT3 group.

siSTAT3 suppressed the inhibition ability of IL-37 on the transcription of TNF- $\alpha$ and IL-1 $\beta$. STAT3 is known as a novel transcription factor for many downstream molecules. Here, we detected whether IL-37 influences TNF- $\alpha$ and IL-1 $\beta$ transcription activity through STAT3. Here, the gene transcription level is relative to GAPDH. Under the state of STAT3 knockdown, compared IL37-NC group to IL37+siSTAT3 group, the transcription level of TNF- $\alpha$ was reduced from 0.46 to 0.29 (48h) in Hela cells(Figure $4 \mathrm{~A}$ ) and from 0.521 to 0.25 in C33A cells (Figure 4B). The transcription level of IL- $1 \beta$ was reduced from 0.35 to 0.24 (48h) in Hela cells (Figure 4C) and from 0.51 to 0.35 in C33A cells (Figure 4D). These results suggested that STAT3 contributed to the regulation of IL-37 on TNF- $\alpha$ and IL-1 $\beta$.

\subsection{STAT3 up regulation restored the cell proliferation and invasion and also the expres- sion of TNF- $\alpha$ and IL- $1 \beta$.}

Transfection of STAT3 expression vector successfully up regulated STAT3 expression. The mRNA level of STAT3 increased by $98 \%$ in Hela cells (Figure $5 \mathrm{~A}$ ) and $110 \%$ in C33A cells (Figure 5B). The protein level of STAT3 increased by $90.71 \%$ in Hela cells (Figure 5C) and $66.08 \%$ in C33A cells (Figure 5D), respectively. Figure 5E and 5F showed that STAT3 gene transfection restored the cell proliferation. Figure $3 \mathrm{G}$ and $3 \mathrm{H}$ showed that STAT3 gene transfection restored the cell invasion.

STAT3 over expression restored the cell proliferation and invasion and increased the transcription of TNF- $\alpha$ and IL- $1 \beta$. In Both Hela cells and C33A cells, IL37+STAT3 groups showed markedly lower inhibition rates of the cell proliferation (Figure 5I and $5 \mathrm{~J}$ ) and cell invasion (Figure 5K and 5L) than that of IL37-NC group. This phenomenon indicated that STAT3 up regulation led the restoration of cell proliferation and invasion. After STAT3 over expression, the proliferation inhibition rates by IL-37 expression were reduced to $11.94 \%$ (24h), $7.56 \%$ (48h) and $10.22 \%$ (72h) (Figure 5I) in Hela cells and 7.14\% (24h), 8.7\% (48h) and 5.26\% (72h) (Figure 5J) in C33A cells. The inhibition rates of invasion were reduced to $7.69 \%$ for Hela cells (Figure 5K) and to $7.14 \%$ for C33A cells at $48 \mathrm{~h}$ (Figure $5 \mathrm{~L}$ ). Thus, the cell proliferation and invasion are close to their normal state following STAT3 over expression. These results inferred that STAT3 up regulation restored the growth ability of tumor cells.

STAT3 up regulation restored the transcription of TNF- $\alpha$ and IL-1 $\beta$. Compared IL37-NC group to IL37+STAT3 group, STAT3 over expression increased TNF- $\alpha$ transcription level from 0.43 to 0.95 (relative to GAPDH) in Hela cells (Figure 6A) and from 0.55 to 
0.89 (relative to GAPDH) in C33A cells (Figure 6B). Compared IL37-NC group to IL37+STAT3 group, STAT3 over expression increased IL- $1 \beta$ transcription
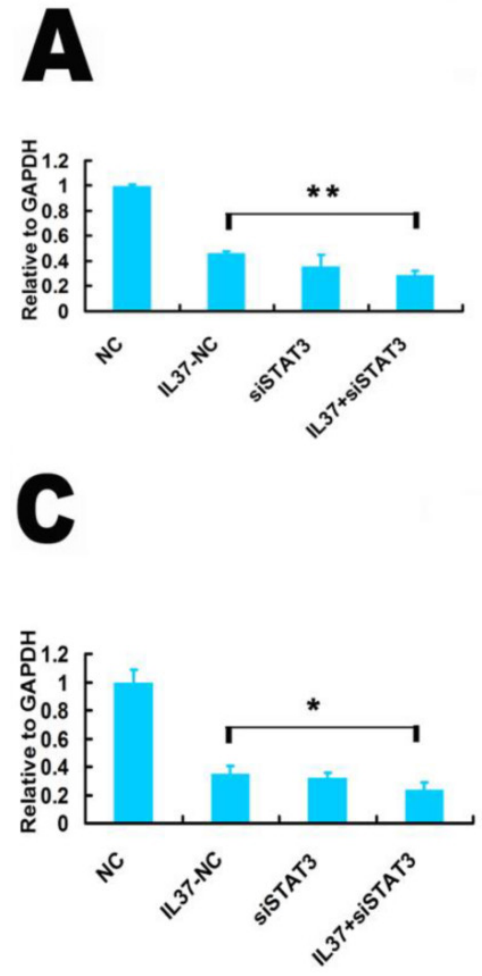

level from 0.42 to 0.92 (relative to GAPDH) in Hela cells (Figure 6C) and from 0.49 to 0.93 (relative to GAPDH) in C33A cells (Figure 6D).

B

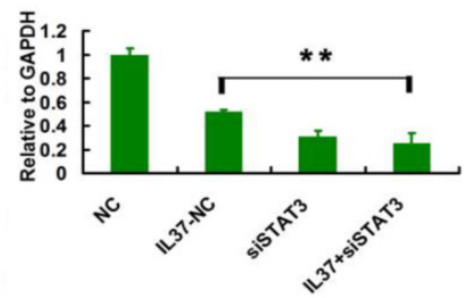

D

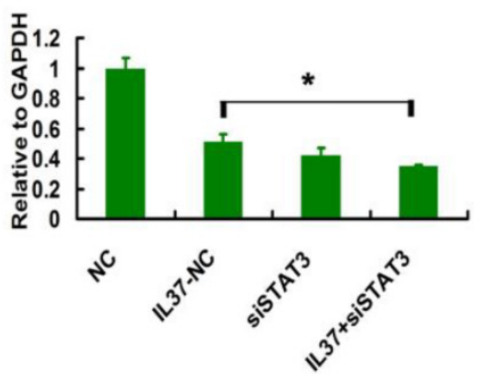

Figure 4. STAT3 siRNA (siSTAT3) transfection reduced the inhibition ability of IL-37 on TNF-aand IL-1ßin Hela and C33A cells. (A, B) siSTAT3 reduced the inhibition ability of IL-37 on TNF-atranscription in Hela cells (A) and in C33A cells (B). (C, D) siSTAT3 reduced the inhibition ability of IL-37 onIL-1 Btranscription in Hela cells (C) and in C33A cells (D). NC: normal control. siSTAT3: STAT3 knockdown group. IL37-NC: IL-37 gene transfection group. IL37+siSTAT3: IL-37 gene transfection with blockage of STAT3 group.

\section{$\mathbf{A}$}

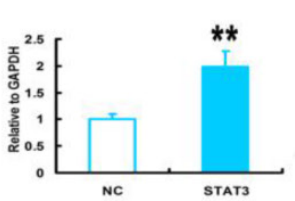

C
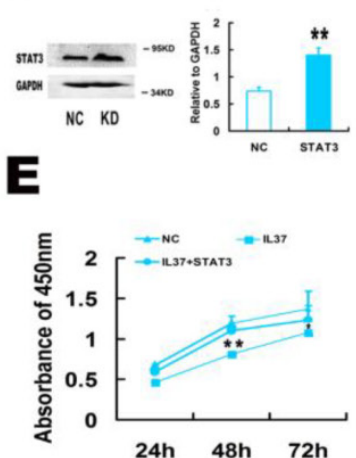

$\mathbf{B}$

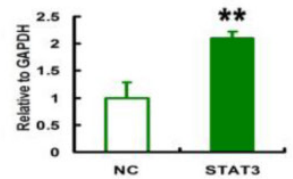

D
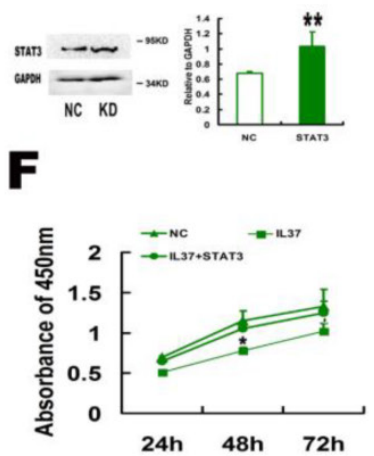

G

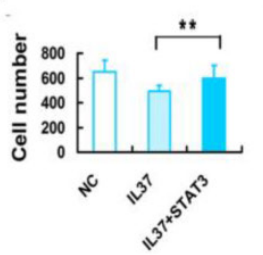

$\mathbf{H}$
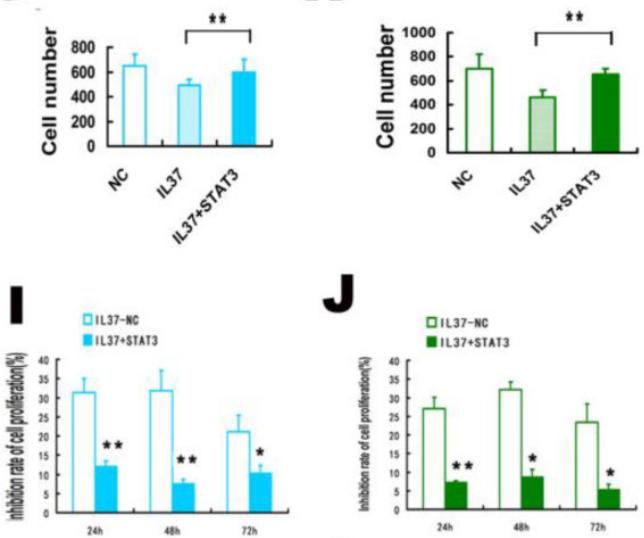

$\mathbf{K}$

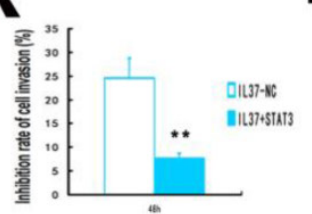

J

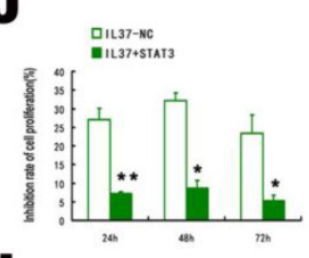

$\mathbf{L}$

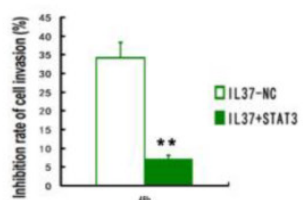

Figure 5. STAT3 up regulation restored the proliferation and invasion ability in Hela and C33A cells. (A, B) STAT3 gene transfection increased the mRNA expression of STAT3 in Hela (A) and C33A (B) cells. (C, D) STAT3 gene transfection increased the protein expression of STAT3 in Hela (C) and C33A (D) cells. (E, F) STAT3 increased the cell proliferation ability Hela (E) and C33A (F) cells. (G, H) STAT3 increased the cell invasion of Hela (G) and C33A (H) cells. (I, J) STAT3 transfection decreased the inhibition rate of proliferation and restored the cell proliferation in Hela $(\mathrm{I})$ and C33A $(\mathrm{J})$ cells. $(\mathrm{K}, \mathrm{L})$ STAT3 transfection decreased the inhibition rate of invasion and restored the cell invasion of Hela $(\mathrm{K})$ and C33A (L) cells. NC: normal control. STAT3: STAT3 up regulation group. IL37-NC: IL-37 gene transfection group. IL37+STAT3: STAT3 and IL-37 gene transfection group. 

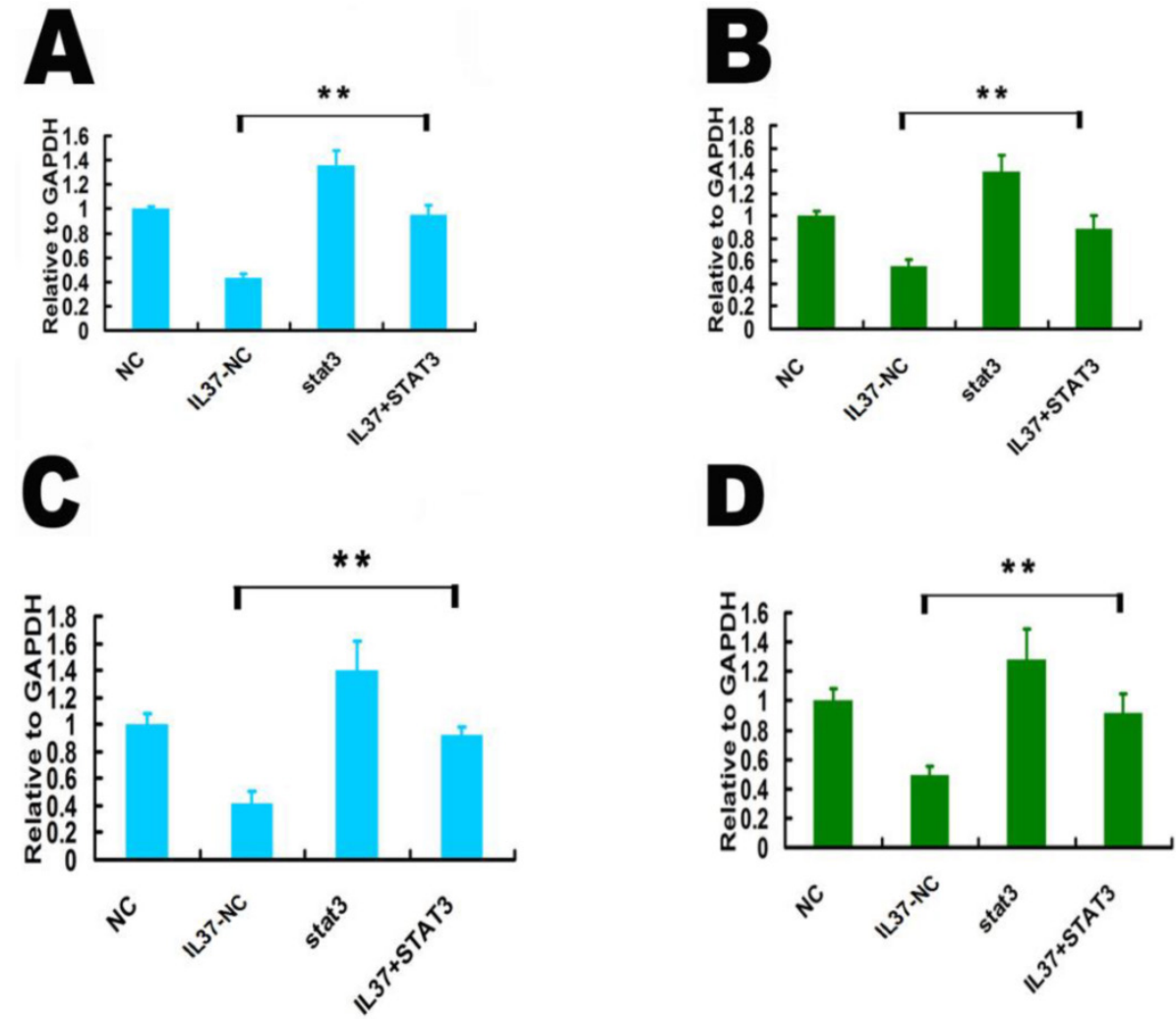

Figure 6. STAT3 up regulation restored the transcription of TNF-and IL-1 $\beta$ in Hela and C33A cells. (A,B) STAT3 over expression increased TNF-atranscription in Hela cells (A) and C33A cells (B). (C,D) STAT3 over expression increased IL-1 $\beta$ transcription in Hela cells (C) and C33A cells (D). NC: normal control. STAT3: STAT3 up regulation group. IL37-NC: IL-37 gene transfection groups. IL37+STAT3: IL-37 and STAT3 gene transfection group.

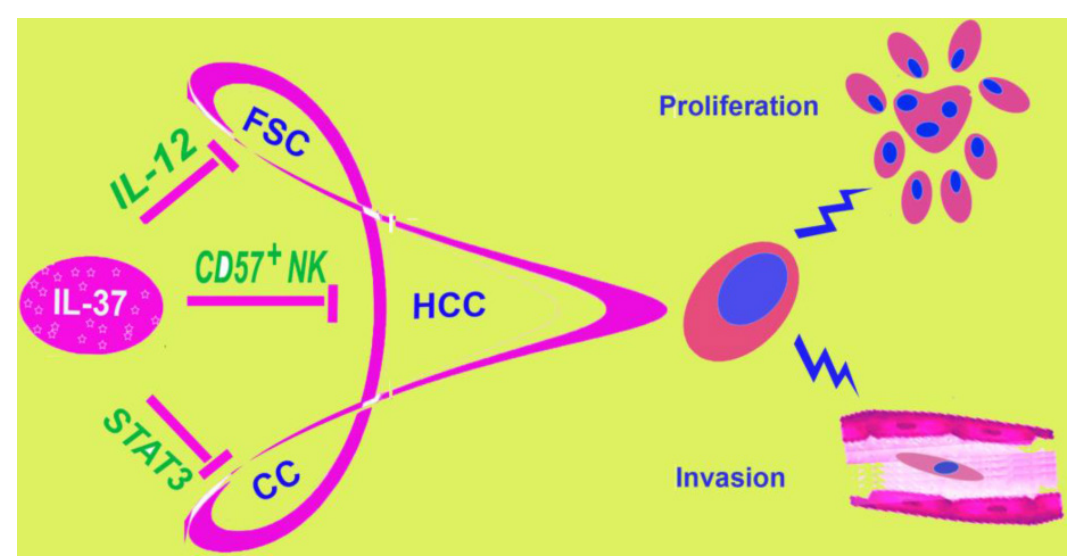

Figure 7. IL-37 is involved in different tumors. IL-37 has been found to play a role in three malignant tumors: fibrosarcoma, hepatocellular carcinoma and cervical cancer, by different molecular mechanisms. This figure showed three different mechanisms of IL-37 regulation on different tumors. FSC, fibrosarcoma; HCC, hepatocellular carcinoma; CC, Cervical Cancer.

\section{Discussion}

During the years, the relationship between IL-37 and tumors remain unclear. Our studies showed a role of IL-37 to inhibit the proliferation and invasion of CC cells, both HPV+ Hela and HPV- C33A. IL-37 has been reported to relate to fibrosarcoma [11] and HCC [12] in previous research (Figure 7). At first glance, it might seem haphazard for IL-37 exploration in the two very different tumors, both of the two studies discussed the mechanism via immune cells.
By contrast, inflammations caused by HPV infection play very important role in cervical cancer. Thus, we detected IL-37 function on CC. IL-37 expression plasmid was transfected into Hela and C33A cells firstly. Once exogenous IL-37 was expressed, the proliferation and invasion of CC cells were inhibited significantly. These results demonstrated our guess. Interestingly, IL-37 showed stronger anticancer ability in HPV+ Hela cells than in HPV- C33A cells, in both proliferation and invasion aspects. The fact implied that this novel gene might have a unique role in virus 
infection. This might correlate with immune responses induced by HPV and needed further exploration.

In this study, signal transducer and activator of transcription 3 (STAT3) was found to be involved in the inhibition of cell proliferation and invasion by IL-37. STAT3 is one of the key molecules in influencing immune responses in tumor [16, 17]. Activated STAT3 increased the cell proliferation and invasion and promoted oncogenic inflammation. Thus, STAT3 is an important target to redirect inflammation for cancer therapy. Our results showed that exogenous IL-37 reduced STAT3 expression at both mRNA and protein levels. STAT3 phosphorylation was down regulated by IL-37. Although this seems not similar to that in immune cells who natively express endogenous protein of IL-37, this molecule might lead to consistent anticancer targets by complex ways: indirect inhibition via regulation of immune responses (like recruiting the natural killer cells) or direct suppression of tumor cells. Furthermore, after trasnfection with siSTAT3, the ability of IL-37 to suppress cell proliferation and invasion was markedly reduced in both Hela and C33A cells. Importantly, STAT3 over expression restored the cell proliferation and invasion and increased the transcription of TNF- $\alpha$ and IL- $1 \beta$. The results indicated that IL-37 suppressed STAT3 expression to influence CC. Additionally, it should be noted that this study has examined only one key downstream signal of cell proliferation and invasion. Notwithstanding its limitation, this study does suggest a new biological function of IL-37 and a potential drug for CC. Next, we will explore comprehensively whether and how IL-37 regulates the apoptosis, cell cycle, autophagy and other aspects of CC.

In conclusion, IL-37 suppressed the cell proliferation and invasion of CC and STAT3 expression and phosphorylation. Importantly, STAT3 knockdown reduced the ability of IL-37 to suppress the cell proliferation and invasion. Thus, IL-37 emerges as a new molecule for CC therapy, which showing good clinical antitumor prospects. Our work demonstrated a new biological function of IL-37 and offered a potential drug for CC prevention and treatment. We believe that an astonishing outpouring of research on the relationship between IL-37 and tumors will be witnessed in the near future.

\section{Acknowledgements}

This study was funded by the National Natural Science Foundation of China (81302244), by the Industrial technology research and development special fund project of Guangdong Province (2013B021800062), by the Social Science and Technology Development Project of Dongguan
(2013108101051, 201410515200188), by the Natural Science Foundation of Guangdong Province (S2012040006383), by the Medical Science and Technology Research Fund of Guangdong province (B2013293) and by the PhD Start-up Fund of Guangdong Medical College (B2011012, B2011015, B2011019).

\section{Conflict of Interests}

The authors declare no conflict of interests.

\section{References}

[1] McNamee EN, Masterson JC, Jedlicka P, et al. Interleukin 37 expression protects mice from colitis. P Natl Acad Sci USA. 2011; 108:16711-6.

[2] Sakai N, Van Sweringen HL, Belizaire RM, et al. IL-37 reduces liver inflammatory injury via effects on hepatocytes and non-parenchymal cells. J Gastroenterol Hepatol. 2012; 27:1609-16.

[3] Luo Y, Cai X, Liu S, et al. Suppression of antigen specific adaptive immunity by IL-37 via induction of tolerogenic dendritic cells. P Natl Acad Sci USA. 2014; 111:15178-83.

[4] Imaeda H, Takahashi K, Fujimoto T, et al. Epithelial expression of interleukin-37b in inflammatory bowel disease. Clin Exp Immunol. 2013; 172: 410-6.

[5] Wan Z, Sun Z, Song F, et al. Down regulated interleukin 37 expression associated with aggravation of intervertebral disc degeneration. Int J Clin Exp Pathol. 2014; 7:656-62.

[6] Wu BW, Zeng QT, Meng K, et al. The potential role of IL-37 in atherosclerosis. Pharmazie. 2013; 68: 857-60.

[7] Moretti S, Bozza S, Oikonomou V, et al. IL-37 Inhibits Inflammasome Activation and Disease Severity in Murine Aspergillosis. PLoS Pathog. 2014; 10:e1004462.

[8] Song L, Qiu F, Fan Y, et al. Glucocorticoid regulates interleukin-37 in systemic lupus erythematosus. J Clin Immunol. 2013; 33:111-7.

[9] Nold MF, Nold-Petry CA, Zepp JA, et al. IL-37 is a fundamental inhibitor of innate immunity. Nature Immunol. 2010; 11:1014-22.

[10] Hanahan D, Weinberg RA. The hallmarks of cancer. Cell. 2000; 100:57-70.

[11] Gao W, Kumar S, Lotze MT, et al. Innate Immunity Mediated by the Cytokine IL-1 Homologue 4(IL-1H4/IL-1F7) Induces IL-12-Dependent Adaptive and Profound Antitumor Immunity. J. Immunol. 2003; 170:107-13.

[12] Zhao JJ, Pan QZ, Pan K, et al. Interleukin-37 mediates the antitumor activity in hepatocellular carcinoma:role for CD57+ NK cells. Sci Rep. 2014; 4:5177-85.

[13] Sushma M, Vamsikrishna B, Babu M M, et al. A review on role of Human Papilomma Virus (HPV) in cervical cancer. Pharma Tutor. 2014; 2: 21-30.

[14] Konnikova L, Simeone MC, Kruger MM, et al. Signal transducer and activator of transcription 3 (STAT3) regulates human telomerase reverse transcriptase (hTERT) expression in human cancer and primary cells. Cancer Res. 2005; 65:6516-20.

[15] Wang S, Pang T, Gao M, et al. HPV E6 induces eIF4E transcription to promote the proliferation and migration of cervical cancer. Febs Lett. 2013; 587: 690-7.

[16] Yu H, Pardoll D, Jove R. STATs in cancer inflammation and immunity: a leading role for STAT3. Nat Rev Cancer. 2009; 9: 798-809.

[17] Yu H, Lee H, Herrmann A, et al. Revisiting STAT3 signalling in cancer: new and unexpected biological functions. Nat Rev Cancer. 2014; 14: 736-46. 